hep-lat/9709009

LSUHE No. 262-1997

OUTP-97-42P

September, 1997

\title{
Magnetic monopole clusters, and monopole dominance after smoothing in the maximally Abelian gauge of SU(2).
}

\author{
${\underline{\text { A. } \text { Hart }^{1}}}^{\text {and M. Teper }}{ }^{2}$ \\ ${ }^{1}$ Department of Physics and Astronomy, Louisiana State University, \\ Baton Rouge. LA 70803 U.S.A. \\ email: hart@rouge.phys.lsu.edu \\ ${ }^{2}$ Theoretical Physics, University of Oxford, \\ 1 Keble Road, Oxford. OX1 3NP U.K. \\ email: teper@thphys.ox.ac.uk
}

(To be published in the proceedings of Lattice 97 - Nucl. Phys. B (Proc. Suppl.). 


\begin{abstract}
In the maximally Abelian gauge of $\mathrm{SU}(2)$, the clusters of monopole current are found to divide into two distinct classes. The largest cluster permeates the lattice, has a density that scales and produces the string tension. The remaining clusters possess an approximate $1 / l^{3}$ number density distribution ( $l$ is the cluster length), their radii vary as $\sqrt{ } l$ and their total current density does not scale. Their contribution to the string tension is compatible with being exactly zero. Their number density can be thought of as arising from an underlying scale invariant distribution. This suggests that they are not related to instantons. We also observe that when we locally smoothen the $\mathrm{SU}(2)$ fields by cooling, the string tension due to monopoles becomes much smaller than the SU(2) string tension. This dramatic loss of Abelian/monopole dominance occurs even after just one cooling step.
\end{abstract}


Magnetic monopole currents [1] are central to the dual superconducter hypothesis for confinement - as seen after Abelian projection [2, 3] to the maximally Abelian gauge (MAG) [4]. Using the unambiguous division of the current into mutually disconnected clusters, we find that only the single largest cluster contributes to the string tension, $\sigma$. We also find that the density of this largest cluster scales well and that the scaling violations seen in the total current density are due to the smaller clusters (see also [5]) which do not contribute to $\sigma$ (see also [6]).

In each configuration we find that one cluster is significantly larger than the rest (on an $L=16$ lattice at $\beta=2.3$ it has mean length 10169 , compared to a mean of only 67 for the second largest). This large cluster fills the entire lattice volume and its length, at fixed $\beta$, is proportional to the volume, $L^{4}$. By contrast the length of the second largest cluster increases much more weakly with $L$ and it is much more localised.

If the total 'length' of magnetic current is $l_{\text {tot }}$, we may use the $S U(2)$ string tension to write the current density in nonperturbatively defined physical units

$$
\rho_{\text {tot }}=\frac{l_{\text {tot }} \cdot a \sqrt{\sigma}}{(L \cdot a \sqrt{\sigma})^{4}}=\frac{l_{\text {tot }}}{L^{4}(a \sqrt{\sigma})^{3}} .
$$

Similarly we define $\rho_{\max }$ for the largest cluster alone. Whilst the total current density shows strong scaling violations in Fig. 1, that of the largest cluster alone shows remarkably good scaling from $\beta=2.3$ to 2.5 .

The time-like monopole current links in a given time slice of the lattice may be treated as a 3d gas of magnetic charges. Moving out from a given charge, we observe charge screening characteristic of a plasma. The screening length for the magnetic current from the largest cluster alone is many times greater than that for the combined smaller clusters. Since the screening length is related to the string tension, this and the scaling behaviour already suggest that the largest cluster plays the dominant rôle in the infrared physics.

The separate contributions to the string tension from the largest cluster, and from the remainder are shown in Table 1. The string tension is consistent with being entirely due 
to the largest cluster; particularly at smaller values of $\beta$ where the distinction in length between the largest and second largest clusters is clearest and the volumes are largest in physical units.

We now calculate the effective radius, $r_{\text {eff }}$, for each cluster, and plot its average value as a function of cluster length, $l$, in Fig. 2. The data fits well a curve $r_{\text {eff }} \sim \sqrt{l}$, with the parameters showing only a weak dependence on $\beta$. This form is reminiscent of the displacement of a particle undergoing a random walk of $l$ steps.

The cluster 'spectrum', $n(l)$, is the mean number of clusters of a given length on a configuration. It is plotted versus $l$ in Fig. 固 and we see that it is close to a power law $n(l)=c / l^{\gamma}$ for values of $l$ where the errors are small (similar to what was seen for current loops [7]). For all of our data $\gamma \in[2.85,3.15]$.

This exponent may be understood as arising from a general scale invariant distribution of objects of radius $r$ in $4 \mathrm{~d}$. Using $r_{\text {eff }} \sim \sqrt{l}$ then predicts $\gamma=3$ :

$$
n(r) d r \sim \frac{d r}{r} \times \frac{1}{r^{4}} \quad \Rightarrow \quad n(l) d l \sim \frac{d l}{l^{3}} .
$$

We recall that a semiclassical instanton of core size $\rho$ in the MAG generates a monopole loop of proportional length within its core (e.g. [8]). More generally, we expect a cluster of radius $\propto \rho$. A scale invariant distribution of core sizes might occur for large $\rho$ if the action freezes out there. This does not explain, however, why it is the smaller clusters that most clearly fall on the power law. Indeed the small instanton distribution is very far from being scale invariant. Thus instantons are not an obvious source for these monopole clusters.

We turn now to our second topic: the fate of monopole/Abelian dominance on cooled or smoothened $\mathrm{SU}(2)$ fields. We recall that the interest in the MAG arises because here alone the $\mathrm{SU}(2)$ string tension is nearly reproduced by the monopole currents (e.g. [9]). This would seem to imply a correlation between the currents and the long range properties of the $\mathrm{SU}(2)$ vacuum. It is interesting, in light of this, to investigate the behaviour of the $\mathrm{SU}(2)$ and monopole string tensions under the application of small, local deformations of 
the vacuum fields.

An ensemble of configurations was prepared using the Wilson action $\mathcal{S}$ and the $\mathrm{SU}(2)$ string tension was calculated. To each configuration we applied a certain number of 'smoothing'(三 'cooling') steps, to yield a new ensemble and the string tension was calculated afresh. Small amounts of such smoothing merely remove the ultraviolet fluctuations in the vacuum. The infrared properties, including the string tension and, by definition, the lattice spacing, are unchanged.

Taking the smoothed ensemble, we fix to the MAG and obtain the monopoles and their contribution to the string tension. To try to minimise the (small) effects of Gribov copies, the procedure was slightly more complicated. The original SU(2) ensemble was gauge fixed and a single smoothing sweep applied. The configurations were then gauge fixed again. (Since one smoothing step is a small perturbation, it might be expected that the number of sweeps necessary to gauge fix the second time would be smaller than the first but this did not seem to be the case.) Additional smoothing proceeded accordingly.

To estimate the string tension, we here present initial results using the effective string tension defined from the square Creutz ratios, $\sigma_{\text {eff }}(r)=-\log \operatorname{Cr}(r, r)$, which approaches the asymptotic string tension for large $r$ (although not necessarily from above on the smoothened fields where 'positivity' no longer holds).

Fig. W shows the monopole string tension after a number of smoothing steps, and the unsmoothed SU(2) value. Even one smoothing sweep causes a large reduction in the monopole string tension. Further smoothing continues to reduce its value, but at a decreased rate.

The infrared properties of the $\mathrm{SU}(2)$ ensemble have not changed (as we see by an explicit calculation of the $\mathrm{SU}(2)$ string tension), yet the monopole physics is very different. This may have implications for the interpretation of monopole (and Abelian) dominance as a connexion between the long range properties of the $\mathrm{SU}(2)$ vacuum and the magnetic currents.

These results agree with independent studies using different techniques [10], but con- 
trast, however, with [11] which used Metropolis cooling (a much less efficient way of removing short distance fluctuations). Further investigation is clearly necessary here.

\section{Acknowledgements}

The work of A.G.H. was supported by the United States Department of Energy grant DE-FG05-91ER40617 and that of M.T. by United Kingdom PPARC grants GR/K55752 and GR/K95338.

\section{References}

[1] T. DeGrand, D. Toussaint, Phys. Rev. D22 (1980) 2478.

[2] S. Mandelstam, Phys. Rept. 23 (1976) 245.

[3] G. 't Hooft, Nucl. Phys. B190 (1981) 455.

[4] A.S. Kronfeld, M.L. Laursen, G. Schierholz, U.J. Wiese, Phys. Lett. B198 (1987) 516.

[5] G.I. Poulis, Phys. Rev. D56 (1997) 161, available as hep-lat/9704006.

[6] J. Stack, S. Neiman, R. Wensley, Phys. Rev. D50 (1994) 3391, available as heplat/9404014.

[7] A. Hart, M. Teper, Nucl. Phys. B (Proc. Suppl.) 53 (1997) 497, available as heplat/9606022.

[8] A. Hart, M. Teper, Phys. Lett. B371 (1996) 261, available as hep-lat/9511016.

[9] G. Bali, V. Bornyakov, M. Müller-Preussker, K. Schilling, Phys. Rev. D54 (1996) 2863, available as hep-lat/9603012.

[10] T. Kovács, Z. Schram, hep-lat/9706012 and these proceedings.

[11] R. Wensley, Nucl. Phys. B (Proc. Suppl.) 47 (1996) 306. 


\begin{tabular}{lcc}
\hline \hline$L=16$ & $\beta=2.3$ & $\beta=2.4$ \\
\hline all: & $0.128(5)$ & $0.067(2)$ \\
largest: & $0.122(5)$ & $0.058(2)$ \\
rest: & $0.000(1)$ & $0.001(1)$ \\
\hline$\%$ curr.: & $75.7(3)$ & $56.8(3)$ \\
\hline \hline
\end{tabular}

Table 1: The monopole string tension, and the proportion of the total current in the largest cluster.

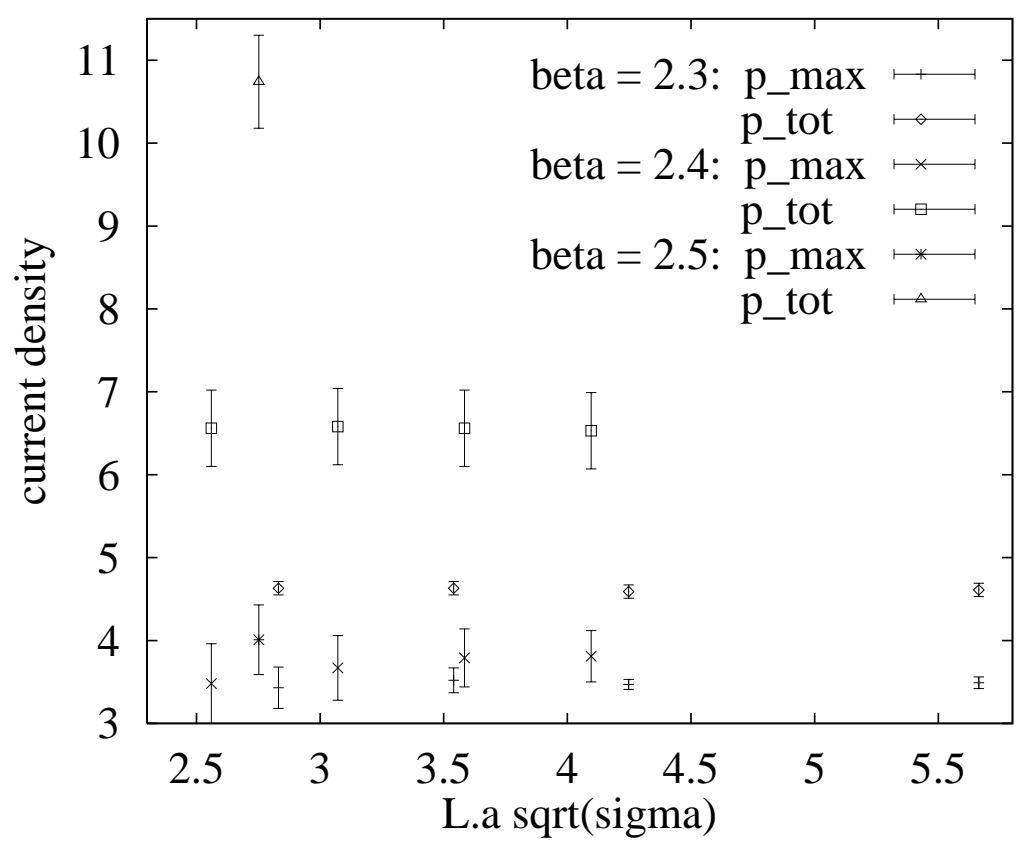

Figure 1: Scaling with the lattice size of the total current density and that of the largest cluster. 


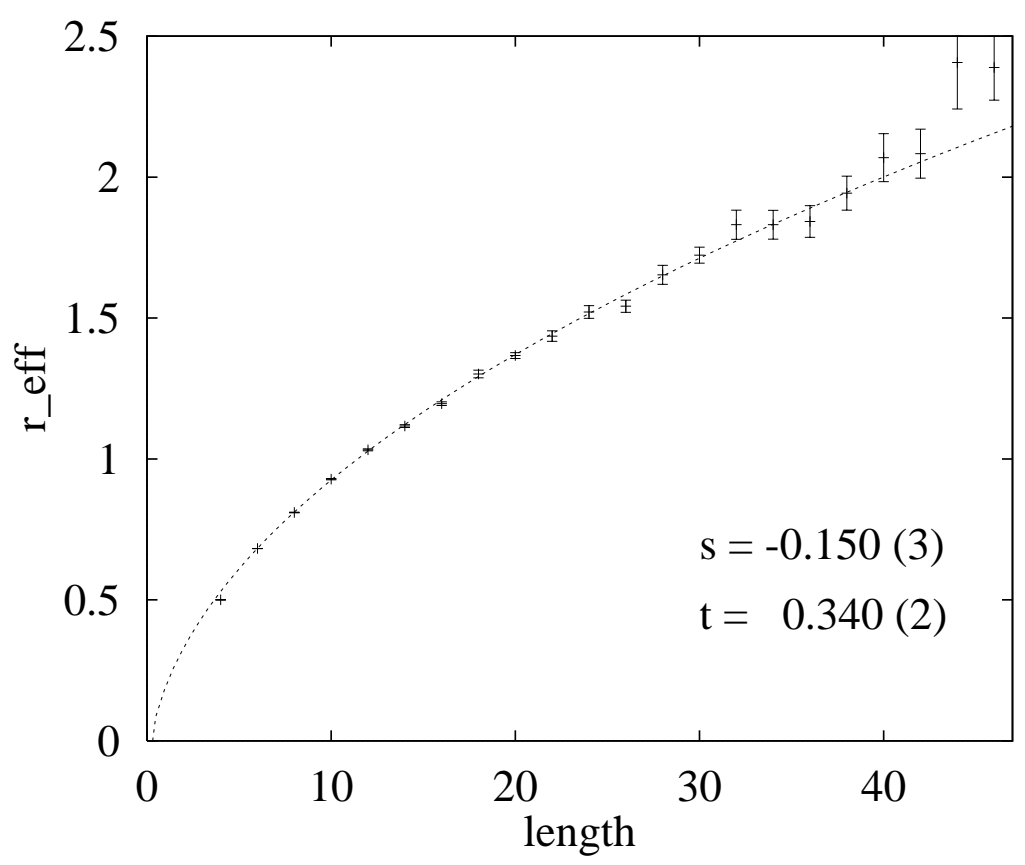

Figure 2: The fit $r_{\text {eff }}=s+t \sqrt{ } l$ at $\beta=2.3$ on $12^{4}$.

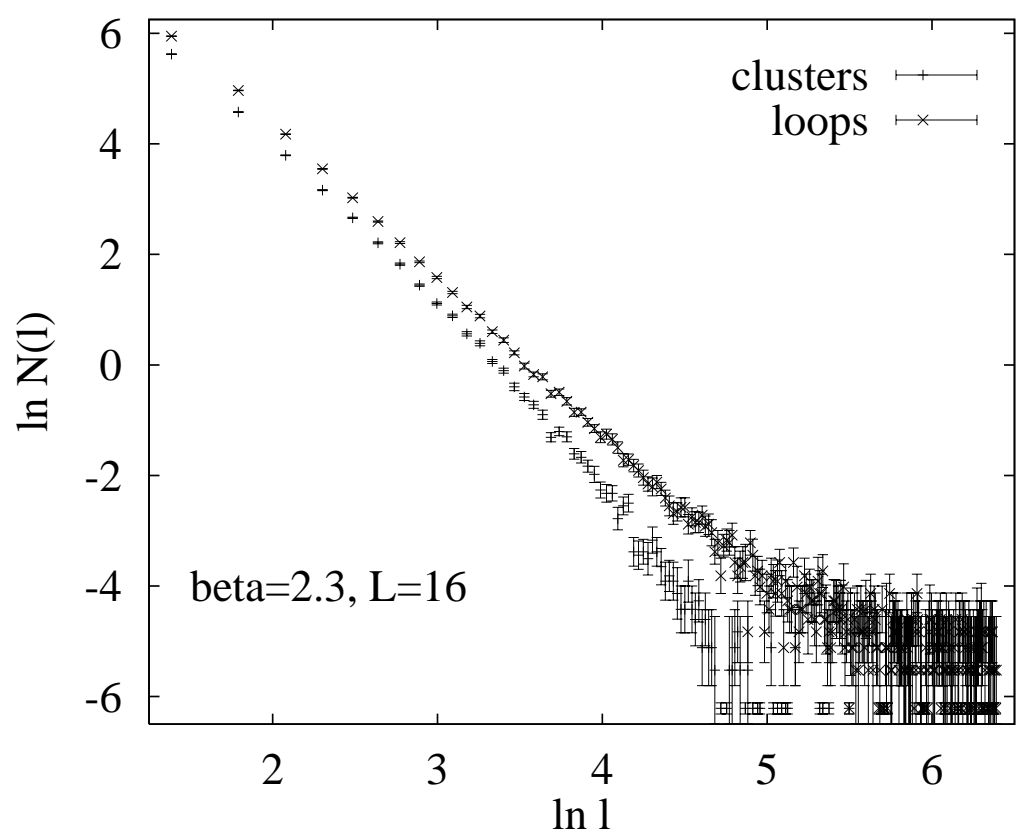

Figure 3: Monopole loop and cluster spectra. 


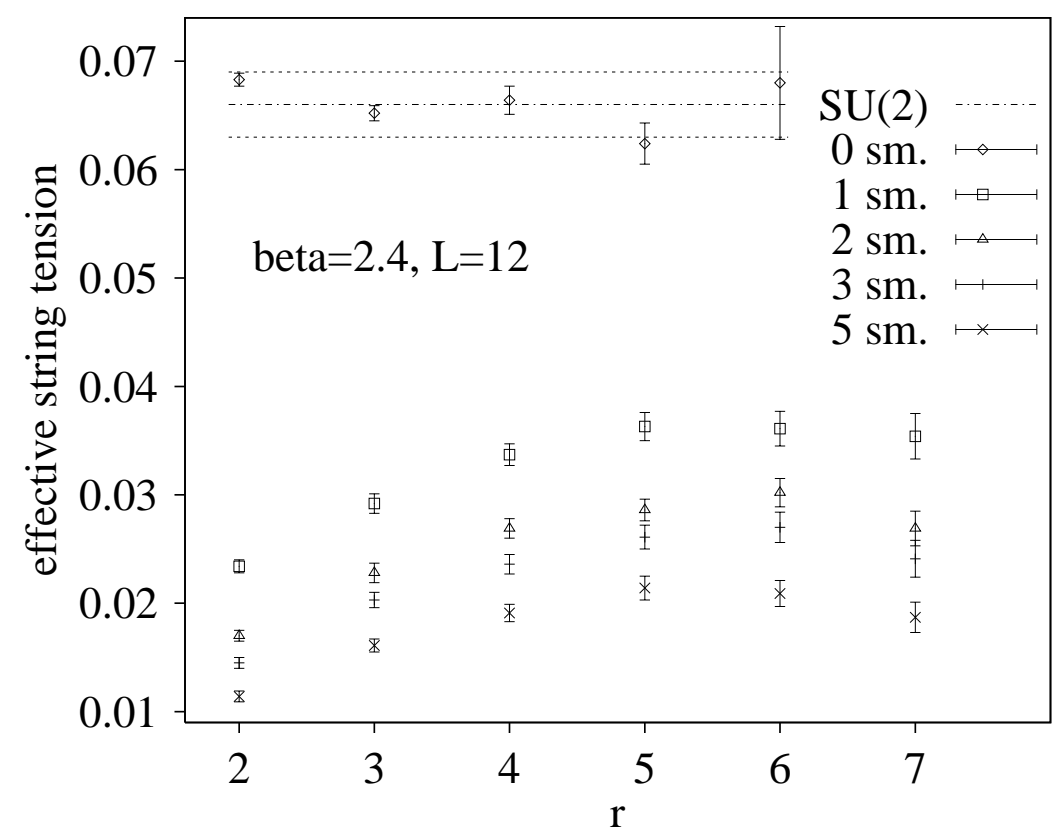

Figure 4: Smoothing and the effective string tension. 